C-A/AP/\#179

November 2004

\title{
Vertical Bump Effect at PHOBOS in Run-4
}

\author{
S.Y. Zhang
}

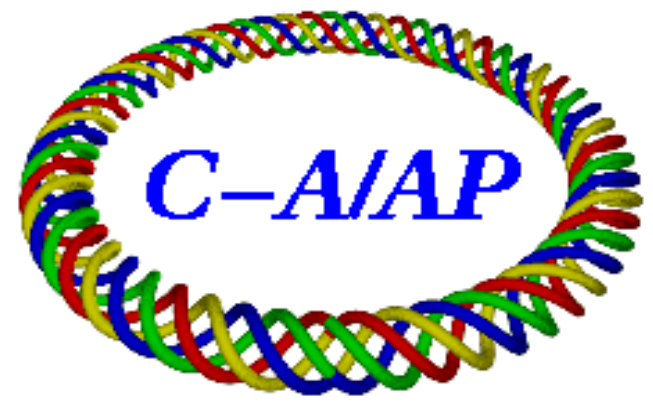

Collider-Accelerator Department Brookhaven National Laboratory Upton, NY 11973 


\title{
Vertical Bump Effect at PHOBOS in Run-4
}

\author{
S.Y. Zhang
}

\begin{abstract}
In the gold operation of RHIC Run 2004, the experiment background of PHOBOS had limited the beam intensity, and hence the luminosity. This background was caused by the pressure rise at the beam rebucketing. In this note, the effect of the vertical orbit bump at the PHOBOS on the rebucketing pressure rise is reviewed. If proven effective, then it may be applied to the Run 2005 copper operation for luminosity improvement.
\end{abstract}

\section{Introduction}

In the gold operation of RHIC Run 2004, the experiment background of PHOBOS had limited the beam intensity, and hence the luminosity. This background was caused by the pressure rise at the beam rebucketing. In this note, the effect of the vertical orbit bump at the PHOBOS on the rebucketing pressure rise is reviewed. Intensive operation studies during the ramps 4480 to 4548, total 31 cases, are analyzed by reviewing E-log, the rebucketing pressure rise, the data of the orbit, DX BPM, and corrector current. Most cases confirm that when a $8 \mathrm{~mm}$ vertical bump was applied at the time of rebucketing, the pressure did not rise. The beam intensity and the bunch peak current at the rebucketing in these ramps are compared with the pressure rise, indicating that it is indeed the bump effect that matter. Several cases of the bump effect after the rebucketing, with the established pressure rise, also show that the vertical bump may reduce the pressure rise significantly. Finally, after the ramp 4548, the vertical bump was kept eliminated until the end of run. Data show that most high intensity ramps during this period had the rebucketing pressure rise. The ramps that did not have the pressure rise 
may because of the low intensity, poor rebucketing process, and may because of the absent of the PHOBOS magnet ramping.

There are a few cases cannot be satisfactorily explained by the bump effect along. Therefore, in the coming Run 2005, beam study will be proposed for better understanding. If this effect is proven still relevant, then it can be applied to copper run for luminosity improvement.

\section{Vertical bump effect at the rebucketing}

Most difficult part of this study is that the data of the vertical orbit, the DX BPM, and the corrector current are far from complete. The data logging of the orbit bump and DX BMP was triggered by separate systems, which are disabled if a 'manual' mode is used. The corrector current logging is only once a minute. The lack of accurate account of the orbit had caused the confusion during that period of operation studies. Making the situation even less clear, many other factors in the play, such as the beam intensity, beam condition, storage RF voltage, rebucketing procedure, etc, are all taking their effect.

In this review, therefore, we focused on the events of orbit and DX BPM just before and right after the rebucketing time. The results are shown in Table 1. The assumption is that if the $8 \mathrm{~mm}$ vertical bump at the PHOBOS was applied at the beam rebucketing, it should help to eliminate the rebucketing pressure rise, whereas if it is not in, pressure rises. If the orbit data right before and after the rebucketing consistently agree with this assumption, then the bump effect is OK, shown in column 8. If the data before the rebucketing is inconsistent, but the one right after the rebucketing is consistent, the status is O. For the cases that no data available at and after the rebucketing time, the DX BPM data is used for the reference. This leaves 19 cases with OK, 9 cases with $\mathrm{O}$, and 3 cases are questionable.

In Table 1, The boldfaces in column 3 indicate the rebucketing pressure rise at PHOBOS. The boldfaces in columns 5, 6, and 7 indicate the data consistent with the bump effect. The corresponding time is shown. 


\begin{tabular}{|c|c|c|c|c|c|c|c|}
\hline 1 & 2 & 3 & 4 & 5 & 6 & 7 & 8 \\
\hline Ramp & Total & Pressr. & Time & Vert. orbit & Vert. orbit & DX & Bump \\
\hline & Int. & Rbkt & Rbkt & Before rbkt & After rbkt & BPM & Effect \\
\hline 4480 & 90.2 & 0.06 & $02: 23: 39$ & $\mathbf{8}[23: 37]$ & $8[23: 42]$ & & $\mathrm{OK}$ \\
\hline 4487 & 95.4 & 6.0 & $23: 10: 37$ & $\mathbf{0}[10: 37]$ & $\mathbf{0}[10: 42]$ & & OK \\
\hline 4492 & 93.0 & 2.3 & $13: 38: 21$ & $5[37: 54]$ & $5[42: 55]$ & & $? 1$ \\
\hline 4493 & 91.9 & 1.21 & $18: 44: 37$ & $7[44: 23]$ & 7 [49:09] & & $? 2$ \\
\hline 4506 & 91.8 & 0.64 & $10: 37: 50$ & $\mathbf{0}[37: 24]$ & $\mathbf{0}[42: 24]$ & & OK \\
\hline 4507 & 89.8 & 0.05 & $17: 28: 14$ & $7[27: 47]$ & $7[32: 47]$ & & $\mathrm{OK}$ \\
\hline 4508 & 90.5 & 0.04 & $00: 13: 31$ & $7[13: 04]$ & $7[16: 44]$ & & OK \\
\hline 4509 & 84.7 & 0.04 & $03: 21: 01$ & $7[20: 34]$ & $7[23: 51]$ & & OK \\
\hline 4510 & 81.5 & 0.04 & 09:01:36 & $7[01: 09]$ & $7[06: 09]$ & & OK \\
\hline 4512 & 81.0 & 1.40 & $18: 54: 38$ & $7[54: 11]$ & $3[56: 27]$ & & $\mathrm{O}$ \\
\hline 4513 & 77.5 & 0.05 & $00: 47: 51$ & $8[47: 24]$ & $8[52: 24]$ & & OK \\
\hline 4522 & 89.7 & 0.86 & $18: 52: 47$ & $\mathbf{0}[52: 19]$ & $\mathbf{0}[53: 44]$ & & $\mathrm{OK}$ \\
\hline 4523 & 93.0 & 1.37 & $00: 15: 33$ & $8[15: 05]$ & None & $\mathbf{0}[19: 54]$ & $\mathrm{O}$ \\
\hline 4525 & 90.3 & 1.24 & $06: 14: 50$ & $2[14: 22]$ & $2[17: 40]$ & & OK \\
\hline 4526 & 99.8 & 2.3 & $13: 14: 17$ & $8[13: 49]$ & $8[18: 49]$ & & $? 3$ \\
\hline 4527 & 90.7 & 0.08 & 19:06:29 & $9[06: 10]$ & $9[09: 48]$ & & OK \\
\hline 4528 & 95.5 & 0.09 & $00: 39: 19$ & $9[38: 52]$ & None & $8[40: 23]$ & OK \\
\hline 4529 & 95.9 & 0.09 & 06:03:04 & $\mathbf{9}[02: 37]$ & $\mathbf{9}[07: 37]$ & & OK \\
\hline 4530 & 97.1 & 0.07 & $11: 41: 13$ & $7[40: 46]$ & $8[42: 44]$ & & OK \\
\hline 4531 & 97.3 & 1.99 & 17:09:14 & $8[08: 48]$ & None & $\mathbf{0}[11: 04]$ & $\mathrm{O}$ \\
\hline 4533 & 88.7 & 0.54 & $00: 03: 55$ & $\mathbf{0}[03: 28]$ & $\mathbf{0}[08: 28]$ & & OK \\
\hline 4534 & 80.2 & 0.46 & $05: 21: 14$ & $\mathbf{0}[20: 48]$ & $\mathbf{0}[23: 48]$ & & OK \\
\hline 4535 & 96.3 & 1.07 & $12: 22: 38$ & $\mathbf{0}[22: 11]$ & $\mathbf{0}[27: 11]$ & & OK \\
\hline 4536 & 94.3 & 1.14 & $17: 33: 03$ & $\mathbf{0}[32: 36]$ & $\mathbf{0}[36: 28]$ & & OK \\
\hline 4537 & 91.2 & 2.51 & $23: 45: 10$ & $7[44: 42]$ & None & $\mathbf{0}[48: 44]$ & $\mathrm{O}$ \\
\hline 4538 & 83.8 & 1.00 & $04: 21: 23$ & $8[20: 55]$ & None & $\mathbf{0}[22: 44]$ & $\mathrm{O}$ \\
\hline 4539 & 96.3 & 1.13 & $09: 40: 37$ & $7[40: 10]$ & None & $\mathbf{0}[41: 40]$ & $\mathrm{O}$ \\
\hline 4540 & 93.3 & 1.09 & $15: 07: 21$ & $8[06: 55]$ & $\mathbf{0}[09: 52]$ & & $\mathrm{O}$ \\
\hline 4544 & 94.7 & 0.99 & $00: 57: 21$ & $7[56: 54]$ & None & $\mathbf{0}[00: 28]$ & $\mathrm{O}$ \\
\hline 4547 & 90.6 & 0.70 & $12: 54: 27$ & $\mathbf{2}[53: 59]$ & $\mathbf{0}[58: 59]$ & & $\mathrm{OK}$ \\
\hline \multirow[t]{2}{*}{4548} & 101.3 & 2.20 & $17: 07: 19$ & $8[06: 52]$ & $0[11: 52]$ & & $\mathrm{O}$ \\
\hline & $10^{9}$ & $10^{-9}$ Torr & & $\mathrm{mm}$ & $\mathrm{mm}$ & $\mathrm{mm}$ & \\
\hline
\end{tabular}

Table 1: Vertical bump effect at PHOBOS. 
It is noticed that there might be a threshold effect, which means that for a given bump, the high intensity beam may still have the rebucketing pressure rise, or, for a given beam intensity, smaller bump might be not sufficient to stop the pressure rise. This way, the questionable cases 4492 and 4526 might be explained: the bump of $5 \mathrm{~mm}$ in 4492 may be not large enough, and the intensity in 4526 , about $100 \times 10^{9} \mathrm{Au}$ ions, might be too high. The case 4493 cannot, however, be explained by the bump effect along.

Among many factors affecting the rebucketing pressure rise, the beam intensity and the sum of bunch peak currents at the rebucketing are probably the most important ones. The peak current is critical for the electron multipacting, and it varies by a factor of 2 during the period. The storage RF voltage applied to the beam rebucketing changed for ramp to ramp, at $4 \mathrm{MV}$ to $4.8 \mathrm{MV}$ for Blue ring, and at $3 \mathrm{MV}$ to $4 \mathrm{MV}$ for Yellow ring. Moreover, the rebucketing procedure was constantly under tunning for improvement, and beam conditions including longitudinal and transverse emittances were not the constant for these ramps.

In Figure 1, the rebuckting pressure rise is compared with the beam intensity, and the sum of peak currents at the rebucketing, respectively. There are general trends for both, indicating fundamental mechanism in electron multipacting, i.e., higher beam intensity and higher peak current help electron multipacting. The ramps without rebucketing pressure rise, total 10, are all confirmed with sufficient vertical bumps. These are 4480, 4507, 4508, 4509, 4510, 4513, 4527, 4528, 4529 and 4530 .

It is noticed that the ramps of 4528, 4529, and 4530 are with high intensity, from $95.5 \times 10^{9}$ to $97.1 \times 10^{9}$ gold ions, which also have highest peak currents from $9.8 \mathrm{~A}$ to $10.6 \mathrm{~A}$. Moreover, these ramps are with 56 bunch mode, which is in general unfavorable for the rebucketing pressure rise caused by the electron multipacting, compared with the 45 bunch mode later adopted. Nevertheless, the rebucketing pressure rise was absent. The effectiveness of applying vertical bumps looks plausible, and it is certainly worth a study.

\section{$3 \quad$ Vertical bump effect after the rebucketing}

The vertical bump effect after the rebucketing, with the established pressure rise, can be shown for the ramps of 4487, 4539 and 4544.

In the ramp of 4487, shown in Figure 2, the DX BPM Bi9 tv3, and Yo10 tv 5 are shown with the PHOBOS pressure. The pressure reduced a little 

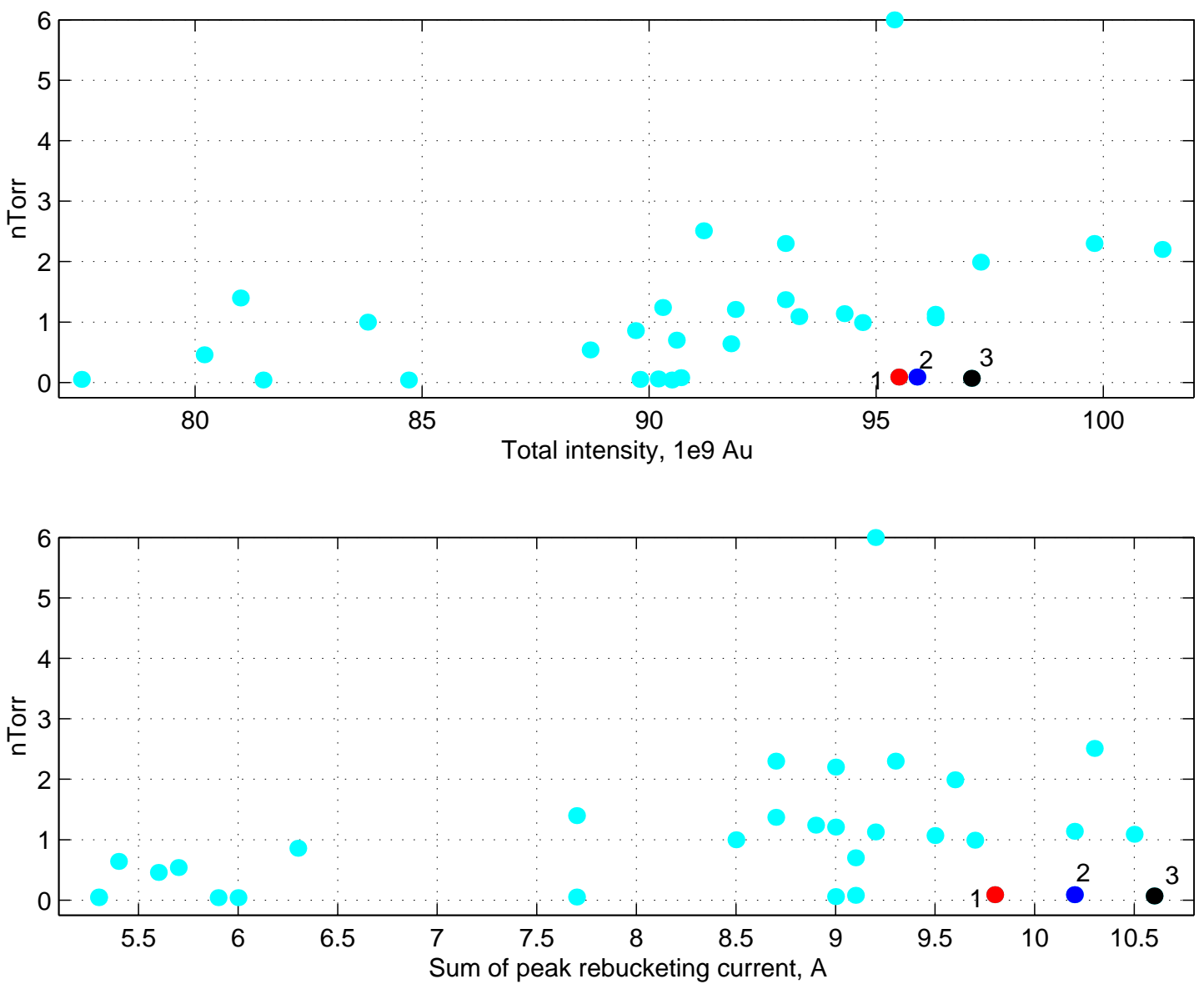

Figure 1: PHOBOS rebucketing pressure rise vs. total beam intensity (top), and the sum of peak currents at the rebucketing (bottom), respectively. There are general trends for both, indicating electron multipacting mechanism. Ramps 4428 (1), 4429 (2), and 4430 (3) have both high intensity and high peak current, but without rebucketing pressure rise. The vertical bump was on for these ramps. 

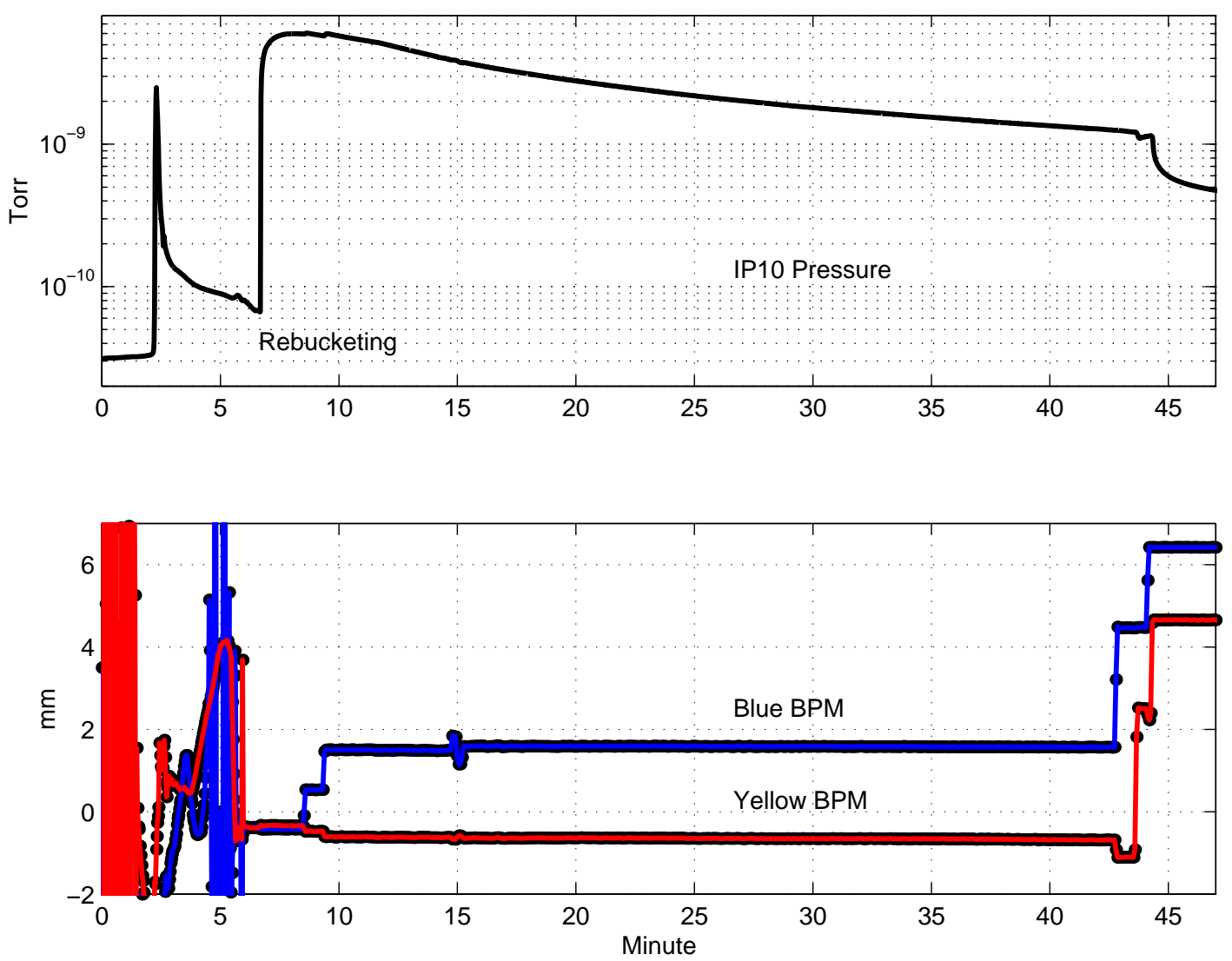

Figure 2: Ramp 4487, PHOBOS rebucketing pressure rise and Blue and Yellow bumps. At the rebucketing, there was no bump, pressure rises. Starting from about 43 minute, the blue bump was raised to $6.5 \mathrm{~mm}$, but a close look shows that it is the yellow bump raised to $4.7 \mathrm{~mm}$ that caused large pressure drop. 
when Bi9 tv3 was raised to $6.5 \mathrm{~mm}$, and it dropped when Yo10 tv5 was raised to $4.7 \mathrm{~mm}$. It looks that in this case, the yellow beam steering was more effective in terms of pressure reduction.

In the ramp of 4539, shown in Figure 3, when Bi9 tv3 raised to $8 \mathrm{~mm}$, the pressure dropped.

In the ramp of 4544, shown in Figure 4, when Bi9 tv3 raised to $9 \mathrm{~mm}$, the pressure dropped. Afterwards, with much lower pressure, the steering effect can still be identified with small pressure variation.

Not all the sudden drop of the rebucketing pressure can be attributed to vertical bumps. In the ramps of 4512 and 4531, when the pressure suddenly reduced, no vertical bump movements were identified.

\section{Ramps without vertical bump}

After the ramp 4548, the activity of setting vertical bump at the PHOBOS stopped, this left more than 100 ramps afterwards without vertical bump. During this period, about half of the ramps were without rebucketing pressure rise, and others had. Also, 45 bunch mode was applied to most ramps, the mitigation effect of the 45 bunch mode was clearly demonstrated compared with the 56 bunch mode. This is consistent with the understanding of the electron multipacting, since that the 45 bunch mode has larger bunch spacing.

Mechanisms helping to eliminate or reduce the rebucketing pressure rise include the low beam intensity, poor rebucketing and hence lower peak current, and the lack of PHOBOS magnet ramping. Analyzing these factors may help to identify the vertical bump effect at the rebucketing pressure rise.

The rebucketing pressure rise of total 57 high intensity ramps are plotted against the total beam intensity in Figure 5. All ramps with highest intensities are included, but most ramps with the total intensity less than $85 \times 10^{9} \mathrm{Au}$ ions are not. Also, a few ramps without any information on the rebucketing peak current are not included.

There is a general trend in the pressure rise with the total intensity, again indicating fundamental electron multipacting mechanism. Most high intensity ramps had rebucketing pressure rise. Only 13 cases were with the pressure below $10^{-10}$ Torr, which are either considered low or without the rebucketing pressure rise. Three of these cases 1 (4587), 2 (4822), and 3 (4945) are without PHOBOS magnet ramping. It is known that for all cases without PHOBOS magnet ramping, rebucketing pressure rise was absent. 

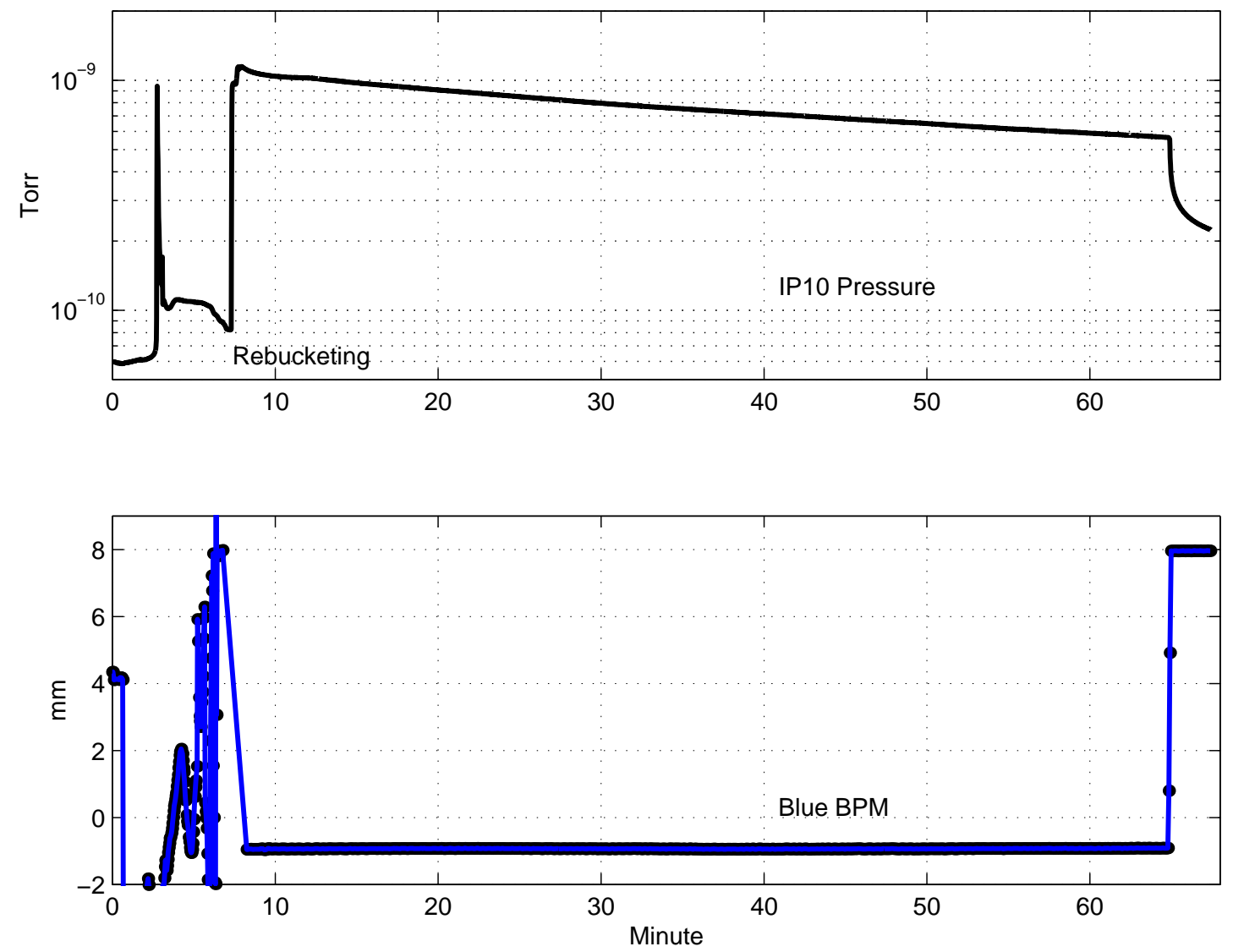

Figure 3: Ramp 4539, PHOBOS rebucketing pressure rise and Blue bump. At the rebucketing, there was no data, but the one at about 8 minute indicating zero bump, therefore, this case is in status O, rather than OK, in Table 1. At about 65 minute, the blue bump was raised to $8 \mathrm{~mm}$, and the pressure dropped drastically. 

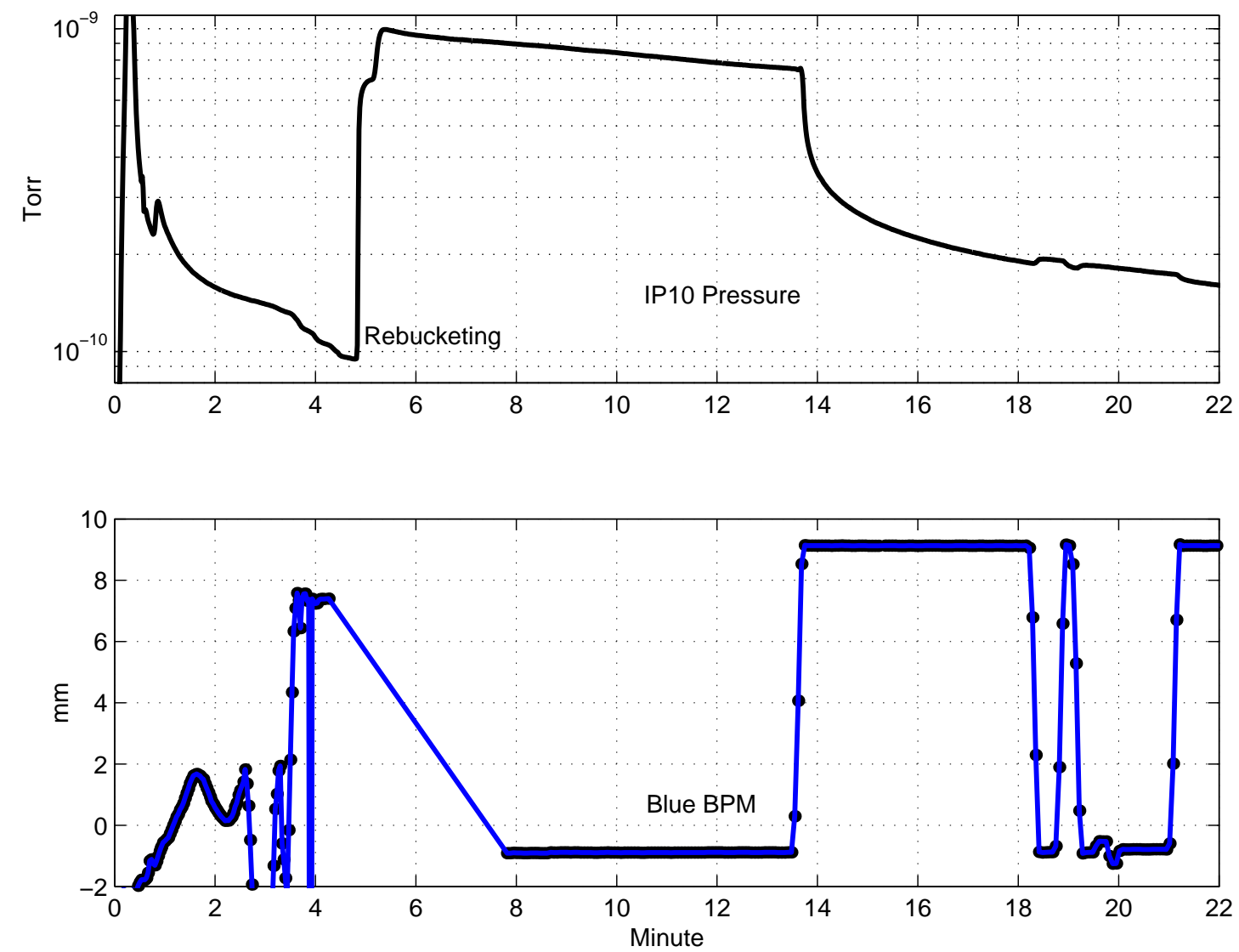

Figure 4: Ramp 4544, PHOBOS rebucketing pressure rise and Blue bump. Again at the rebucketing, there was no data, but the one at about 8 minute indicating zero bump, therefore, this case is also in status O, rather than OK, in Table 1. At about 13.5 minute, the blue bump was raised to $9 \mathrm{~mm}$, and the pressure dropped drastically. Between 18 to 22 minutes, the pressure still responded to the bump change. 


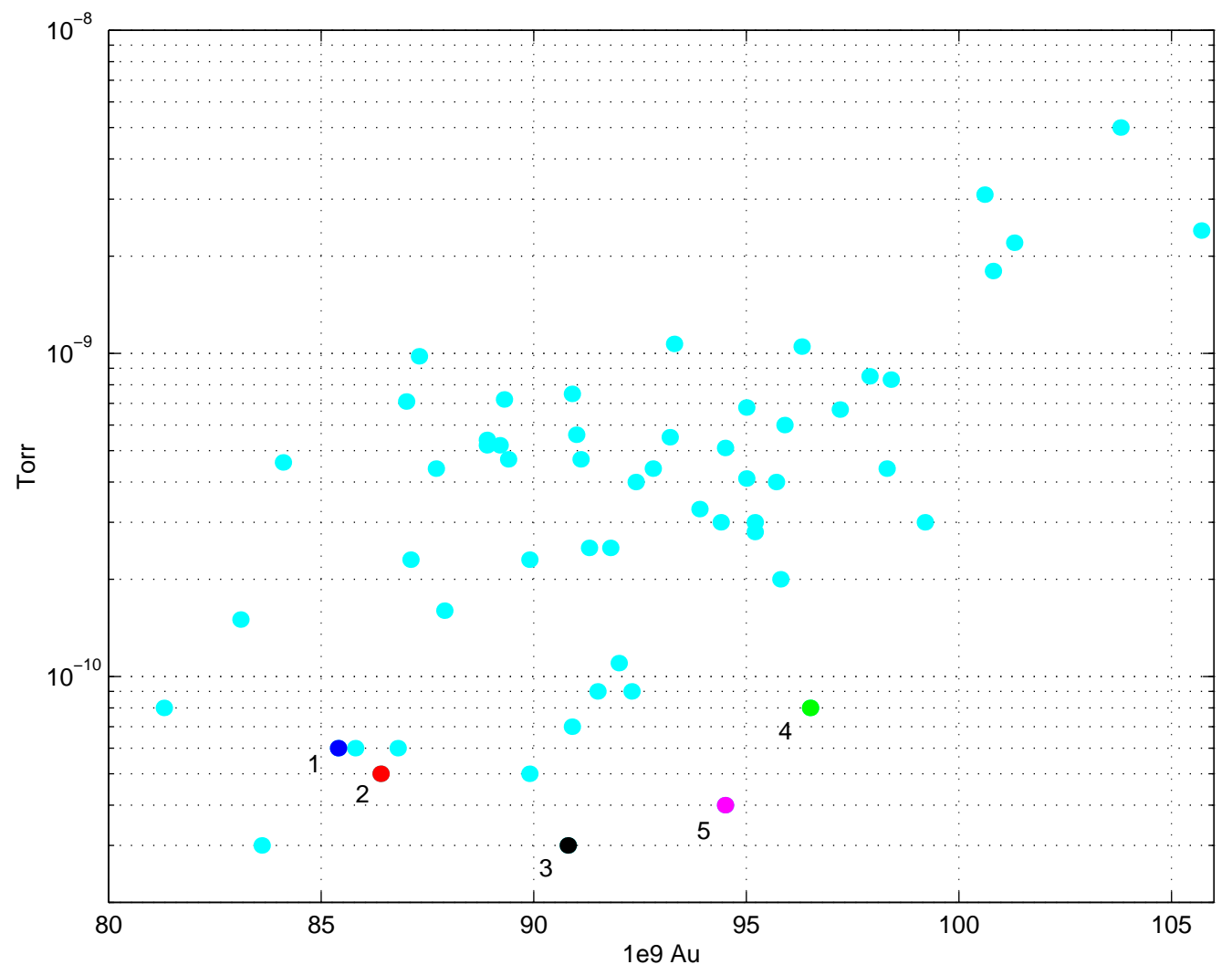

Figure 5: Rebucketing pressure rise of 57 high intensity ramps after 4548, without vertical bumps, vs. total beam intensity. There is a trend of the pressure rise compared with the total beam intensity, indicating electron multipacting mechanism. Only 13 cases had pressure below $10^{-10}$ Torr, which are either considered low or without the rebucketing pressure rise. Ramps 4587 (1), 4822 (2), and 4945 (3) were without PHOBOS magnet ramping (all cases without magnet ramping were without rebucketing pressure rise in Run 2004). The ramp 4622 (4) was with very poor rebucketing, the sum of peak currents was only $5.2 \mathrm{~A}$, compared with overall average of $9.33 \mathrm{~A}$. The case of 4713 (5) was with sum of peak currents of 8.9 A. This case probably cannot explained by the vertical bump effect along. 
The case 4 (4622), had very low peak current of $5.2 \mathrm{~A}$, compared with the overall average of $9.33 \mathrm{~A}$. The case 5 (4713) had sum of peak current $8.9 \mathrm{~A}$, a little lower than average. It is not clear why the rebucketing pressure rise did not occur for this case.

\section{Mechanism and discussion}

Most cases shown above agree that during the Run 2004, the PHOBOS vertical bump had affected the rebucketing pressure rise. The mechanism is, however, not very well understood.

One possibility is that the vertical bump at the IP10 may have affected the secondary electrons' lifetime. If the secondary electrons stay longer, the electron multipacting intensity threshold can be reduced. This is especially critical for the RHIC warm section electron cloud. One assumes that the beam halo scraping generated positive ions can help electrons to stay, as suggested in [1]. It is not impossible that the vertical bump could affect this halo scraping. This mechanism is still under discussion.

Another possibility is that a simulation has shown that with $8 \mathrm{~mm}$ orbit deviation the electron multipacting at PHOBOS is delayed a little, with a slightly lower saturation level [2]. This mechanism is, however, unable to explain the RHIC electron cloud at warm sections, which has much larger bunch spacing than other machines, and the non-uniform distribution in the ring.

A beam study is desirable in the coming copper run to help for better understanding. To prepare for the study, the orbit and BPM logging need to improve, at least the data around the beam rebucketing should be logged.

Even the PHOBOS vertical bump effect can be proved for Run-4, it is not guaranteed that it will repeat itself in Run-5. A good example is that the rebucketing pressure rise at the interaction region IP2 was absent in Run-4, whereas in Run-3 the IP2 was very similar to IP10 in both transition and rebucketing pressure rises.

\section{Acknowledgment}

The author would like to thank A. Drees, W. Fischer, V. Ptitsyn, and T. Roser for numerous helpful discussions. 


\section{References}

[1] S.Y. Zhang, RHIC Vacuum Pressure Bump, C-A/AP/67, Jan. 2002.

[2] G. Rumolo and W. Fischer, Observations on background in PHOBOS and related electron cloud simulations, C-A/AP/146, March 2004. 